\title{
Letter to Editor: COVID-19 and Mental Health of People with Autism Spectrum Disorder and Their Families; What Can Be Done?
}

\author{
Mahta Alsadat Aarabi (iD) ${ }^{1}$, Kianoush Abdi (iD ${ }^{1,{ }^{*}}$ and Mohammad Saeed Khanjani (iD) ${ }^{2}$ \\ ${ }^{1}$ Department of Rehabilitation Management, University of Social Welfare and Rehabilitation Sciences, Tehran, Iran \\ ${ }^{2}$ Department of Counseling, University of Social Welfare and Rehabilitation Sciences, Tehran, Iran \\ "Corresponding author: Department of Rehabilitation Management, University of Social Welfare and Rehabilitation Sciences, Tehran, Iran. Email: k55abdi@yahoo.com
}

Received 2020 December 18; Revised 2020 December 23; Accepted 2020 December 23.

Keywords: COVID-19, Coronavirus, Autism, Autistic, Mental, Mental Health, Psycho

\section{Dear editor,}

Autism spectrum disorder is one of the most common developmental disorders, which depends on a variety of factors, including specific characteristics defined by social interactions, restricted interest, repetitive behaviors (1), stereotypes, and perceptual and expressive language (2).

As a highly contagious and infectious disease, COVID19 is caused by the new coronavirus. The new virus was announced as a worldwide pandemic by the World Health Organization on March 11, 2019. This virus infects healthy people who are in close contact with sick ones. The coronavirus is mainly spread by saliva droplets and nasal discharge through coughing or sneezing. These droplets spread on equipment and surfaces around the patient. Other people get infected with COVID-19 by touching these objects or contaminated surfaces and then touching their eyes, mouth, or nose. That is why it is very important to follow respiratory etiquette. The best way to control and reduce the rate of transmission is to raise awareness about the nature of the virus, the disease it causes, and the ways of spread. The World Health Organization suggests a selfprotection protocol that involves washing hands, using alcohol-based rubs frequently, and not touching the face area (3). Unfortunately, the spread of the virus has disrupted the function of all global communities, but in general, children are infected with a milder form of the virus (4).

The intense fear of the coronavirus, known as "corona phobia", has led to the outbreak of a variety of psychological manifestations in different strata of society (5). The COVID-19 pandemic has required us to make very rapid and far-reaching changes in daily life and society at large. The effects of this pandemic will be greater and more significant on people with autism (6). Many governments have enacted restrictive laws and regulations to prevent the spread of the coronavirus. Staying at home is one of those activities for parents and children. In these circumstances, caring for some children, such as children with autism spectrum disorders who have special needs, can be challenging for families and caregivers (7). The prevalence of the virus, especially in people who suffer from previous mental health conditions, can lead to severe distress in the psyche. People with autism spectrum disorder are especially at risk because of their greater vulnerability to unpredictable and intricate changes (8). Depression, having difficulty with coping with changes, and interacting with others are some of the challenges people with autism face with most of the time. However, in the midst of this pandemic, all these challenges are getting intensified (9). This disease increases anxiety and distress in people with autism and their families. Repeated adherence to health tips and advice, as well as practicing isolation and social distancing, may disrupt the daily routine of people with disabilities and cause distress in them (10).

It is clear that in such special critical situations as this pandemic, we will witness dramatic changes in the mental health of people with autism spectrum disorders. Some of the most common changes in their mental health include sleep disorders, aggression, irritability, belligerence, screaming, and stereotyped behaviors (11). In fact, they are much more likely to suffer from depression, anxiety, stress, and symptoms of PTSD when compared to people without such disabilities. As mentioned, in addition to individuals themselves, the mental health of their families is also affected by COVID-19. Many parents report low mood and high anxiety $(12,13)$.

Finally, despite that inadequate research has been published on people with autism and their families during the 
coronavirus pandemic, experts believe that COVID-19 has had a profound effect on them, and it will worsen the mental health of people with autism, especially because of the uncertainty (6).

Considering the poor mental health status of people with autism and their families, the following recommendations are provided in the COVID-19 pandemic condition:

Demanding authorities to identify and pay special attention to the complex needs of this group and make plans to prevent further psychological damage to these people and their families due to the unfavorable conditions of the coronavirus epidemic and its aftermath.

Creating models by relevant institutions to prevent and intervene in psychosocial crises for people with autism and their families, with participation and assistance of government resources, health professionals in various fields, and the families to better deal with psychosocial problems and complications caused by the coronavirus.

Providing fully practical and fluent psychological solutions and instructions from reputable psychology centers and psychologists for people with autism, their families, and NGOs to pay close attention to the changes and try to cope better with the coronavirus pandemic situation.

Providing support for families, such as expert consultation on how to meet their children's psychological and educational needs, and setting up appropriate resources and tasks for home learning by the government section.

Creating teleconsultation and telerehabilitation protocols for people with autism spectrum disorder and their families, and providing training courses to clarify these protocols by holding specialized meetings on this issue.

Allocating funds to provide the psychological services needed by these individuals and their families with the follow-up of the relevant bodies by the government.

Increasing media support and providing programs to improve the mental health of these individuals and their families through a variety of social networks, as well as popular government and non-government media.

It is expected that conducting sufficient research provides enough evidence for policymakers and intervening managers to provide psychological services for people with autism spectrum disorder and their families during and even after the outbreak of the coronavirus pandemic, to improve their mental health status and ultimately enhance their quality of life by implementing thoughtful measures.

\section{Footnotes}

Authors' Contribution: None declared by the authors.
Conflict of Interests: The authors declare that they have no competing interests.

Funding/Support: This research did not receive any specific grant from funding agencies in the public, commercial, or not-for-profit sectors.

\section{References}

1. American Psychiatric Association. Diagnostic and statistical manual of mental disorders (DSM-5®). American Psychiatric Pub; 2013.

2. Pennington ML, Cullinan D, Southern LB. Defining autism: variability in state education agency definitions of and evaluations for autism spectrum disorders. Autism Res Treat. 2014;2014:327271. doi: 10.1155/2014/327271. [PubMed: 24987527]. [PubMed Central: PMC4060325].

3. WHO. Coronavirus overview. 2020. Available from: https://www.who. int/health-topics/coronavirus\#tab=tab_1.

4. Lim T, Tan MY, Aishworiya R, Kang YQ. Autism Spectrum Disorder and COVID-19: Helping Caregivers Navigate the Pandemic. Ann Acad Med Singap. 2020;49(6):384-6. [PubMed: 32712636].

5. Dubey S, Biswas P, Ghosh R, Chatterjee S, Dubey MJ, Chatterjee $S$, et al. Psychosocial impact of COVID-19. Diabetes Metab Syndr. 2020;14(5):779-88. doi: 10.1016/j.dsx.2020.05.035. [PubMed: 32526627]. [PubMed Central: PMC7255207].

6. Cassidy SA, Nicolaidis C, Davies B, Rosa SDR, Eisenman D, Onaiwu MG, et al. An Expert Discussion on Autism in the COVID-19 Pandemic. Autism Adulthood. 2020;2(2):106-17. doi:10.1089/aut.2020.29013.sjc.

7. Narzisi A. Handle the Autism Spectrum Condition During Coronavirus (COVID-19) Stay At Home period: Ten Tips for Helping Parents and Caregivers of Young Children. Brain Sci. 2020;10(4). doi: 10.3390/brainsci10040207. [PubMed: 32244776]. [PubMed Central: PMC7226467].

8. Colizzi M, Sironi E, Antonini F, Ciceri ML, Bovo C, Zoccante L. Psychosocial and Behavioral Impact of COVID-19 in Autism Spectrum Disorder: An Online Parent Survey. Brain Sci. 2020;10(6). doi:10.3390/brainsci10060341. [PubMed: 32503172]. [PubMed Central: PMC7349059].

9. Patel JA, Badiani AA, Nielsen FBH, Assi S, Unadkat V, Patel B, et al. COVID-19 and autism: Uncertainty, distress and feeling forgotten. Public Health Pract. 2020;1. doi: 10.1016/j.puhip.2020.100034. [PubMed Central: PMC7392884].

10. Fuld S. Autism Spectrum Disorder: The Impact of Stressful and Traumatic Life Events and Implications for Clinical Practice. Clin Soc Work J. 2018;46(3):210-9. doi: 10.1007/s10615-018-0649-6. [PubMed: 30100640]. [PubMed Central: PMC6061115].

11. Carmenate Rodríguez ID, Rodríguez Cordero A. Psychological impact on children with autism spectrum disorder during covid-19 confinement. Multimed. 2020;24:690-707.

12. Asbury K, Fox L, Deniz E, Code A, Toseeb U. How is COVID-19 Affecting the Mental Health of Children with Special Educational Needs and Disabilities and Their Families? J Autism Dev Disord. 2020. doi: 10.1007/s10803-020-04577-2. [PubMed: 32737668]. [PubMed Central: PMC7393330].

13. Nisticò V, Gambini O, Faggioli R, Demartini B. The psychological impact of COVID-19 among a sample of Italian individuals with High Functioning Autism Spectrum Disorder. Res Square. 2020. doi: 10.21203/rs.3.rs-47184/v1. 